\title{
Asymmetric Meckel Cave Enlargement: A Potential Marker of PHACES Syndrome
}

\author{
(D).N. Wright and (D). Wycoco
}

\begin{abstract}
BACKGROUND AND PURPOSE: PHACES syndrome is a complex of morphologic abnormalities of unknown cause and includes posterior fossa abnormalities; head and neck infantile hemangiomas; arterial, cardiac, and eye anomalies; and sternal or abdominal wall defects. Accurate identification of the syndrome is important for optimal treatment. The purpose of this study was to investigate the incidence of asymmetric Meckel cave enlargement, a potential novel imaging marker, in a population of patients referred for evaluation of possible PHACES syndrome.

MATERIALS AND METHODS: Eighty-five patients referred for neuroimaging evaluation of possible PHACES syndrome were identified and stratified on the basis of their ultimate clinical PHACES diagnosis categorization into PHACES, possible PHACES, or not PHACES. MR imaging studies were subsequently reviewed for the presence or absence of unilateral Meckel cave enlargement, with the reviewer blinded to the ultimate PHACES syndrome categorization.

RESULTS: Twenty-five of 85 patients (29\%) were ultimately categorized as having PHACES or possible PHACES according to consensus guidelines. Asymmetric Meckel cave enlargement was present in $76 \%(19 / 25)$ of these patients and in $82 \%(19 / 23)$ of only those patients with definite PHACES. This finding was present in none of the 60 patients determined not to have PHACES syndrome. In 7/19 patients (37\%) with this finding, subtle MR imaging abnormalities consistent with PHACES were missed on the initial MR imaging interpretation.
\end{abstract}

CONCLUSIONS: Asymmetric Meckel cave enlargement was a common feature of patients with PHACES in our cohort and may serve as a novel imaging marker. Increased awareness of this imaging feature has the potential to increase the diagnostic accuracy of PHACES.

ABBREVIATIONS: IAC = internal auditory canal; PHACES = posterior fossa abnormalities; head and neck infantile hemangiomas; arterial, cardiac and eye anomalies; and sternal or abdominal wall defects

$\mathbf{P}$ HACES syndrome (Online Mendelian Inheritance in Man No. 606519; omim.org) is a complex of morphologic abnormalities of unknown cause and includes posterior fossa abnormalities; head and neck infantile hemangiomas, often in a segmental distribution; arterial, cardiac, and eye anomalies; and sternal or abdominal wall defects. ${ }^{1}$ Accurate recognition of the syndrome is important to identify the potentially increased risk associated with the treatment of infantile hemangiomas in the setting of underlying arterial or cardiac pathology, ${ }^{2,3}$ as well as to initiate careful surveillance and aggressive intervention for potential speech and language delays related to posterior fossa anoma-

Received October 6, 2016; accepted after revision January 17, 2017.

From the Department of Radiology (J.N.W.), University of Washington and Seattle Children's Hospital, Seattle, Washington; and Department of Neurological Intervention and Imaging (V.W.), Alterna Wellness Center, Nedlands, Western Australia. Please address correspondence to Jason N. Wright, MD, M/S MA.7.220, Seattle Children's Hospital, PO Box 5371, Seattle, WA 98105; e-mail: jnixon@uw.edu

三 Indicates article with supplemental on-line table.

http://dx.doi.org/10.3174/ajnr.A5140 lies. ${ }^{4,5}$ There is increasing consensus that the stigmata of PHACES may be subtler than previously thought, and new diagnostic criteria are being considered. We have observed that asymmetric Meckel cave enlargement is a frequent neuroimaging finding in patients with PHACES syndrome and may serve as an easily recognizable marker that can improve diagnostic sensitivity.

\section{MATERIALS AND METHODS}

Following Seattle Children's Hospital institutional review board approval, we retrospectively identified 93 patients referred for MR imaging of the head and neck due to concern for PHACES syndrome between 1994 and 2016, based on the presence or history of large $(>5 \mathrm{~cm})$ or segmental head and neck hemangiomas $(n=92)$ or sternal clefting $(n=1)$. Imaging protocols varied but generally included contrast-enhanced MR imaging of the brain and timeof-flight MRA of the head and neck. In all cases, a coronal fluidsensitive sequence (T2-weighted, STIR, or steady-state free precession [balanced fast-field echo, FIESTA, or CISS]) was included in the protocol. Five patients were excluded due to lesions other 


\begin{tabular}{lcccc}
\hline & All PHACES & Definite PHACES & Possible PHACES & Not PHACES \\
\hline No. (\% total) & $25(29)$ & $23(27)$ & $2(2)$ & $60(71)$ \\
Female (No.) (\% category) & $21(84)$ & $19(82)$ & $2(100)$ & $50(83)$ \\
Age (mean \pm SD) & $20 \pm 39 \mathrm{mo}$ & $22 \pm 40 \mathrm{mo}$ & $1 \pm 0.5 \mathrm{mo}$ & $10 \pm 17 \mathrm{mo}$ \\
Age (range) (median) & 5 days to $14 \mathrm{yr}(4 \mathrm{mo})$ & 5 days to $14 \mathrm{yr}(5 \mathrm{mo})$ & $1-2 \mathrm{mo}(1.5 \mathrm{mo})$ & $1 \mathrm{mo}$ to $7 \mathrm{yr}(4 \mathrm{mo})$ \\
Meckel cave enlargement (No.) (\% category) & $19(76)$ & $19(82)$ & $0(0)$ & $0(0)$ \\
Posterior fossa anomalies (No.) (\% category) & $15(60)$ & $15(65)$ & $0(0)$ & $0(0)$ \\
Facial hemangioma (No.) (\% category) & $24(96)$ & $23(100)$ & $1(50)$ & $60(100)$ \\
Arterial anomalies (No.) (\% category) & $21(84)$ & $20(90)$ & $1(50)$ & $0(0)$ \\
Cardiac anomalies (No.) (\% category) & $13(52)$ & $0(52)$ & $0(0)$ & $0(0)$ \\
Eye anomalies (No.) (\% category) & $0(0)$ & $0(0)$ & $1(50)$ & $0(0)$ \\
Sternal or midline abdominal anomalies (No.) & $1(4)$ & & & $0(0)$ \\
(\% category) & & & & \\
\hline
\end{tabular}

than hemangiomas identified on MR imaging, and 3 additional patients were excluded due to lack of available clinical records.

We then stratified the remaining 85 patients on the basis of their ultimate clinical PHACES diagnosis categorization within the published 2009 consensus criteria into PHACES, possible PHACES, or not PHACES. ${ }^{1}$ Current diagnostic criteria are as previously published by Metry et al. ${ }^{1}$ Work-up included a combination of dermatologic evaluation, ophthalmologic examination, cardiology consultation including echocardiography, and structural MR imaging evaluation of the head and neck.

We subsequently reviewed all MR imaging studies for the presence or absence of asymmetric unilateral Meckel cave enlargement, blinded to the ultimate PHACES syndrome categorization. Particular attention was paid to the coronal fluid-sensitive sequences at the level of the sella turcica, which highlighted the comparative volumes of the bilateral Meckel caves. "Presence" was defined as obvious subjective asymmetric enlargement based on visual assessment, with an estimated volume ratio of approximately 1:1.5 used as a cutoff. Our goal was to evaluate the utility of this novel imaging finding in a clinically applicable manner requiring no advanced morphometric analysis for implementation. Minimal asymmetry was not considered sufficient for positivity.

\section{RESULTS}

Of the 85 included patients evaluated by MR imaging for suspicion of PHACES syndrome, 25 patients (29\%) were ultimately categorized as having PHACES $(n=23)$ or possible PHACES $(n=2)$ according to consensus guidelines. Mean and median ages for the PHACES cohort were 20 and 4 months, respectively. Eighty-four percent were female, in line with prior published reports. ${ }^{6}$ Summary statistics for all patients are presented in the Table.

All patients with PHACES presented with large or segmental head or neck hemangiomas, excepting 1 patient (patient 4) categorized as having possible PHACES, who was evaluated for sternal clefting noted at birth and who subsequently developed a left facial segment 3-distribution hemangioma of $<5 \mathrm{~cm}$. Additional diagnostic criteria for PHACES present in each patient are provided in the On-line Table.

Asymmetric Meckel cave enlargement was present in 76\% (19/ 25) of all patients with PHACES (Fig 1); the finding was present in $82 \%(19 / 23)$ of patients with definite PHACES. Patients with Meckel cave involvement were more likely to have a facial segment 1 or 2 distribution of facial hemangiomas, while patients without were more likely to have a facial segment 3 or cervicothoracic distribution, though overlap occurred in both directions. Five patients (patients 2, 5, 11,20, and 23) demonstrated internal auditory canal (IAC) hemangiomas, and 1 patient (patient 23) had a Meckel cave hemangioma, all associated with ipsilateral Meckel cave enlargement.

Of the patients with unilateral Meckel cave enlargement, all enlargements were ipsilateral to the facial or head and neck hemangioma. Sixty-eight percent (13/19) had associated ipsilateral cerebellar hypoplasia, and 58\% (11/19) had dysplastic cerebellar clefting associated with the hypoplasia. This finding is compared with $60 \%(15 / 25)$ with ipsilateral cerebellar hypoplasia in all patients with PHACES, and 48\% (12/25) with associated dysplastic clefting. In all cases, the cerebellar hemispheric abnormalities were ipsilateral to both the hemangioma and the asymmetrically enlarged Meckel cave.

Eighty-nine percent (17/19) of patients with Meckel cave enlargement had craniocervical vascular anomalies, most with arterial dysplasia. This finding is comparable with $84 \%$ (21/25) noted in all patients with PHACES in our cohort. When present, arterial abnormalities were always ipsilateral to the side of the facial hemangioma and Meckel cave enlargement, though additional contralateral abnormalities occurred in a large minority of cases (41\%) (Fig 2).

Major cardiac or arch anomalies were present in 21\% (4/19) of patients with asymmetric Meckel cave enlargement, compared with a slightly higher $28 \%$ (7/25) of all patients with PHACES in our cohort.

Excepting strabismus related to lid or orbital involvement by facial hemangiomas, ocular anomalies described in PHACES were not identified in any patient on imaging or fundoscopic examination.

Of note, in 7 of the 19 patients (37\%) with asymmetric Meckel cave enlargement, there were subtle MR imaging abnormalities of the posterior fossa, intracranial vessels, or aortic arch and cervical vessels that were missed on the initial MR imaging interpretation (patients 1, 2, 3, 6, 8, 14, and 17). One of these patients (patient 3) was nevertheless diagnosed with PHACES prospectively on the basis of cardiac abnormalities identified on echocardiography. The 6 additional patients were not prospectively identified and were only retrospectively diagnosed with PHACES during our review. One of these patients (patient 1) was initially imaged before the original description of PHACES by Frieden et al in $1996 .{ }^{7}$

Sixty of the 85 patients (71\%) evaluated by MR imaging were 


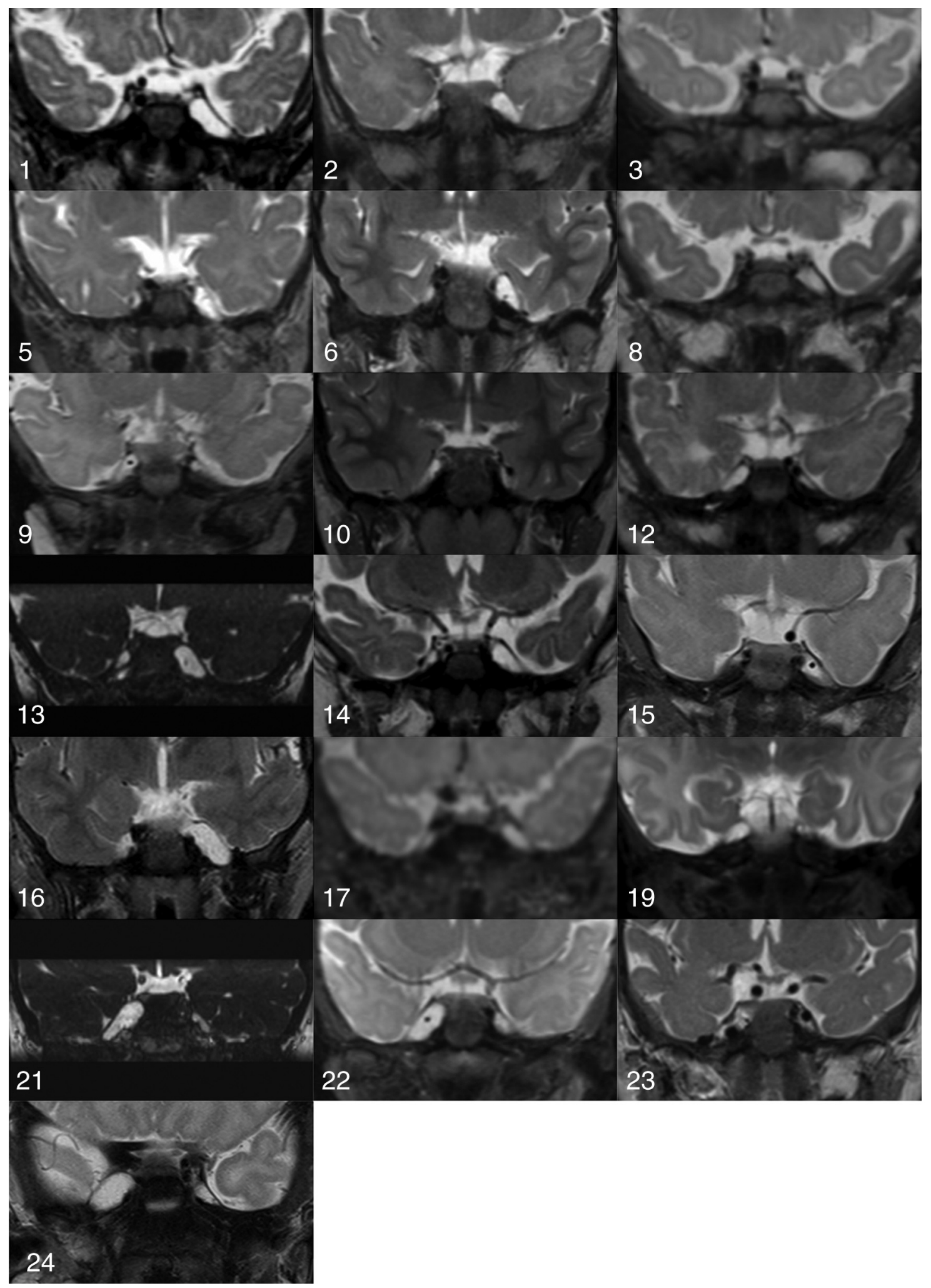

FIG 1. Coronal fluid-sensitive MR images through the bilateral Meckel caves of all patients with asymmetric Meckel cave enlargement. The vascular structure in the enlarged Meckel cave in patients 6, 9, 15, and 22 represents an ipsilateral aberrant ophthalmic artery arising from the basilar artery. The vascular structure in the enlarged right Meckel cave in patient 23 (Fig 2) represents an ectatic persistent trigeminal artery supplying the distal internal carotid artery.

ultimately determined not to have PHACES syndrome. Of these patients, asymmetric ipsilateral Meckel cave enlargement was not identified in any patient.

\section{DISCUSSION}

A subset of patients with large or segmental head and neck hemangiomas will have associated morphologic abnormalities that 

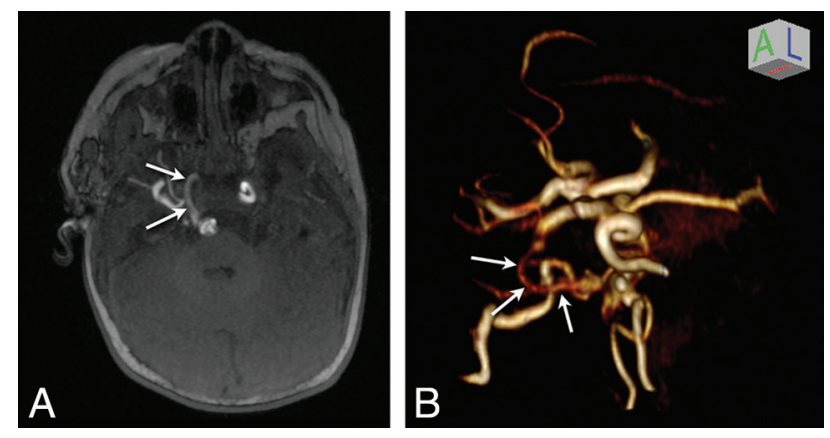

FIG 2. A persistent right trigeminal artery in a 1-year-old girl (patient 23) with PHACES syndrome. A, Axial maximum-intensity-projection 3D time-of-flight image demonstrates a persistent right trigeminal artery (white arrows). Note that the contralateral left cavernous segment internal carotid artery is tortuous. $B$, Volume-rendered 3D timeof-flight reconstruction demonstrates the persistent trigeminal artery (white arrows) connecting the right cavernous segment of the ICA to the tortuous and ectatic basilar artery. Note that the distal right ICA is aplastic proximal to the cavernous segment and is reconstituted via collateral vessels arising from external carotid artery branches.

have fallen under the rubric of PHACES, a neurocutaneous syndrome of uncertain etiology. In our series, $29 \%$ of patients referred for MR imaging evaluation for suspicion of PHACES were ultimately diagnosed with PHACES or possible PHACES per consensus guidelines, in line with the overall prevalence reported in prior studies. ${ }^{1,6}$

Thorough and accurate neuroimaging evaluation is one of the mainstays of complete evaluation for such patients, with MR imaging of the brain and MRA of the head and neck recommended in all cases. In addition to evaluating the distribution and extent of proliferative phase infantile hemangiomas, these studies may reveal abnormalities of the posterior fossa, cervicocranial arteries, globes, or aortic arch and great vessels. These findings are important for optimal risk assessment before initiation of $\beta$ blocker therapy for hemangiomas, ${ }^{2,3}$ as well as to cue neurodevelopmental surveillance for possible associated speech and language delays related to posterior fossa anomalies. ${ }^{4,5}$

In this study, we have demonstrated that the finding of asymmetric Meckel cave enlargement ipsilateral to the facial hemangioma is a common feature of PHACES, present in $76 \%$ of patients. This imaging finding can serve as a useful and conspicuous marker for the syndrome. When applied as an independent diagnostic criterion to our full patient cohort, the finding demonstrated a sensitivity of $76 \%$, a specificity of $100 \%$, a positive predictive value of $100 \%$, a negative predictive value of $91 \%$, and an accuracy of $93 \%$ in predicting a clinical diagnosis of PHACES or possible PHACES syndrome.

In no case was asymmetric Meckel cave enlargement an isolated intracranial finding of PHACES. However, greater than onethird of our patients with Meckel cave enlargement had additional subtle stigmata of PHACES that were missed on the initial evaluation. These may have been more easily or accurately identified with a heightened pretest probability for PHACES associated with asymmetric Meckel cave enlargement. Thus, recognition of this finding could increase the diagnostic sensitivity of neuroimaging for PHACES syndrome. If these additional intracranial findings had been prospectively made in the setting of asymmetric Meckel cave enlargement, the diagnostic sensitivity of MR imaging/MRA for PHACES would have increased by $24 \%$ in our cohort.

Meckel cave enlargement as a finding in PHACES syndrome has only rarely been described in the literature to date, to our knowledge, and is not an abnormality that is currently included in the consensus criteria for PHACES. Given the incidence of the finding in our cohort, this omission likely reflects under-reporting. Oza et $\mathrm{al}^{8}$ described the finding of unilateral Meckel cave prominence in 3 of the 16 patients in their series. The finding was subsequently described in 2 additional case reports. ${ }^{9,10}$ Unilateral Meckel cave enlargement is also demonstrated in Figs 6 and 7 in the text Vascular Lesions of the Head and Neck: Diagnosis and Management by Persky et al, ${ }^{11}$ Figs 1 and 2 from the article by Judd et al, ${ }^{12}$ and Fig 5 from the article by Meltzer et al. ${ }^{13}$ Furthermore, some authors have described an association of arachnoid cysts and PHACES, 1,2,6,8,12,14 and an asymmetric Meckel cave could conceivably be confused with a middle cranial fossa arachnoid cyst, as was the case in one of our patients (patient 21).

The etiology of unilateral Meckel cave enlargement ipsilateral to the facial hemangioma and posterior fossa anomalies in PHACES syndrome is not definitely known. One plausible explanation derives from the theory that PHACES is caused by aberrant or deficient migration of the cephalic neural crest in a metameric distribution. ${ }^{15}$ Neural crest cells and paraxial mesoderm derived from the rhombencephalic metamere contribute to the formation of the skull base; trigeminal nerve ganglia; and facial bones, soft tissues, and blood vessels. Meckel cave enlargement may therefore represent a component of skull base dysplasia secondary to a postzygotic mutation or early prenatal insult in this territory. A similar theory was advanced to explain the relatively high incidence of enlarged IACs seen in PHACES syndrome observed by Meltzer et al. ${ }^{13}$

Alternatively, unilateral enlargement of Meckel cave may be the result of direct expansion secondary to an extant or previously involuted Meckel cave hemangioma. Judd et $\mathrm{al}^{12}$ reported on the association between PHACES and intracranial hemangiomas. Most commonly described in the cerebellopontine angle or IAC, hemangiomas centered in or extending to Meckel cave both have been described in the literature ${ }^{12,13}$ and noted in our series. One of the patients described by Judd et al demonstrated an enlarged Meckel cave containing a hemangioma on initial imaging (Fig $2 \mathrm{C}$ in Judd et al), which then progressed to isolated Meckel cave enlargement following hemangioma involution (Fig $2 E$ in Judd et al), supporting a mechanical etiology of the enlargement.

Metzler et al $^{13}$ similarly raised the possibility of a causal association between IAC hemangioma and IAC enlargement. This was based on the increased prevalence of IAC hemangiomas within the enlarged IACs in children when imaged at younger than 1 year of age, compared with children older than 1 year of age at initial imaging, in whom the hemangiomas were presumed to have previously involuted. Given that Meckel cave hemangioma was present in only 1 of 19 patients with Meckel cave enlargement in our cohort, with a median age of 4 months at imaging, it is unlikely that mechanical enlargement can adequately explain the etiology of this finding in all patients in our cohort.

Limitations of our study included the retrospective nature of the analysis and the relatively small sample size. Also, a subset of 
patients with PHACES may possibly present without obvious cutaneous stigmata. These patients would likely have been missed by our diagnostic algorithm, and any data regarding the prevalence of asymmetric Meckel cave enlargement may not be applicable to this population of patients.

\section{CONCLUSIONS}

Asymmetric Meckel cave enlargement was a common feature of patients with PHACES and possible PHACES in our cohort and may serve as a conspicuous marker for the syndrome. Increased awareness of this imaging feature has the potential to increase the diagnostic accuracy of the neuroimaging evaluation for PHACES in the setting of large or segmental facial hemangiomas.

\section{REFERENCES}

1. Metry D, Heyer G, Hess C, et al; PHACE Syndrome Research Conference. Consensus Statement on Diagnostic Criteria for PHACE Syndrome. Pediatrics 2009;124:1447-56 CrossRef Medline

2. Siegel DH, Tefft KA, Kelly T, et al. Stroke in children with posterior fossa brain malformations, hemangiomas, arterial anomalies, coarctation of the aorta and cardiac defects, and eye abnormalities (PHACE) syndrome: a systematic review of the literature. Stroke 2012;43:1672-74 CrossRef Medline

3. Metry D, Frieden IJ, Hess C, et al. Propranolol use in PHACE syndrome with cervical and intracranial arterial anomalies: collective experience in 32 infants. Pediatr Dermatol 2013;30:71-89 CrossRef Medline

4. Tangtiphaiboontana J, Hess CP, Bayer M, et al. Neurodevelopmental abnormalities in children with PHACE syndrome. J Child Neurol 2013;28:608-14 CrossRef Medline

5. Brosig CL, Siegel DH, Haggstrom AN, et al. Neurodevelopmental outcomes in children with PHACE syndrome. Pediatr Dermatol 2016;33:415-23 CrossRef Medline

6. Metry DW, Haggstrom AN, Drolet BA, et al. A prospective study of PHACE syndrome in infantile hemangiomas: demographic features, clinical findings, and complications. Am J Med Genet A 2006; 140:975-86 Medline

7. Frieden IJ, Reese V, Cohen D. PHACE syndrome: the association of posterior fossa brain malformations, hemangiomas, arterial anomalies, coarctation of the aorta and cardiac defects, and eye abnormalities. Arch Dermatol 1996;132:307-11 Medline

8. Oza VS, Wang E, Berenstein A, et al. PHACES association: a neuroradiologic review of 17 patients. AJNR Am J Neuroradiol 2008;29: 807-13 CrossRef Medline

9. Arora SS, Plato BM, Sattenberg RJ, et al. Adult presentation of PHACES syndrome. Interv Neuroradiol 2011;17:137-46 CrossRef Medline

10. Rosmaninho A, Machado S, Bastos-Leite AJ, et al. PHACE syndrome: a new case report. Eur J Dermatol 2011;21:289-90 CrossRef Medline

11. Persky MS, Waner M, Blei F, et al. Vascular Lesions of the Head and Neck: Diagnosis and Management. New York: Thieme; 2015:chap 6

12. Judd CD, Chapman $P R$, Koch B, et al. Intracranial infantile hemangiomas associated with PHACE syndrome. AJNR Am J Neuroradiol 2007;28:25-29 Medline

13. Meltzer DE, Robson CD, Blei F, et al. Enlargement of the internal auditory canal and associated posterior fossa anomalies in PHACES association. AJNR Am J Neuroradiol 2015;36:2159-62 CrossRef Medline

14. Hartemink DA, Chiu YE, Drolet BA, et al. PHACES syndrome: a review. Int J Pediatr Otorhinolaryngol 2009;73:181-87 CrossRef Medline

15. Krings T, Geibprasert S, Luo CB, et al. Segmental neurovascular syndromes in children. Neuroimaging Clin N Am 2007;17:245-58 CrossRef Medline 